\title{
Elastic Property Simulation of Nano-particle Reinforced Composites
}

\author{
Jiawei $\mathrm{HE}^{1, \text { a }}$, Yong $\mathrm{WANG}^{2, \mathrm{~b}^{*}}$, and Yanan $\mathrm{WANG}^{3, \mathrm{c}}$ \\ ${ }^{1}$ Tianjin Key Laboratory of Refrigeration Technology, Tianjin University of Commerce, Tianjin \\ 300134, China \\ ${ }^{2}$ School of Mechanical Engineering, Tianjin University of Commerce, Tianjin 300134, China \\ ${ }^{3}$ School of Engineering, Deakin University, Waurn Ponds campus, Geelong, Victoria 3216, Australia

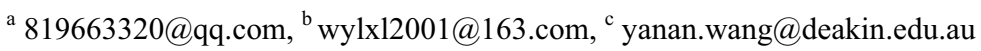

\begin{abstract}
A series of numerical micro-mechanical models for two kinds of particle (cylindrical and discal particle) reinforced composites are developed to investigate the effect of microstructural parameters on the elastic properties of composites. The effects of both the degree of particle clustering and particle's shape on the elastic mechanical properties of composites are investigated. In addition, single particle unit cell approximation is good enough for the analysis of the effect of averaged parameters when only linear elastic response is considered without considering the particle clustering in particle-reinforced composites.
\end{abstract}

Keywords: 3D FE modeling, Elastic Properties, Particle-reinforced composites, Interface effect, Clustering.

\section{Introduction}

Polymer materials reinforced with nano-particles (e.g., nanospheres, nanotubes, nanorods, or nanoplatelets) have received tremendous attention in both scientific and industrial communities due to their extraordinary enhanced properties. The addition of even small amount of particles to a polymer can result in composites materials with significantly improved properties.

In the past few years, research has been focused on the studies of nano-reinforced mechanism for optimization of the microstructure of nanocomposites materials, and some analytical formulas were developed based on micromechanics models by considering the microstructure of nanocomposites observed from experiments. For example, Ji et al. proposed a three-phase model including matrix, interfacial region and fillers and used the model to calculate the tensile modulus of polymer nanocomposites [1]. Brune and Bicerano compared the tensile and compressive elastic moduli of nanoplatelets reinforced composites and predicted the effects on modulus of incomplete exfoliation and imperfect alignment of nanoplatelets [2]. Fornes and Paul used the theories of Halpin-Tsai and Mori-Tanaka to evaluate the effects of filler geometry, stiffness, and orientation and compared the model

*Corresponding author: wylx12001@163.com 
predictions with experimental morphological and mechanical property data [3]. Luo and Daniel developed a three-phase model including epoxy matrix, exfoliated clay nanolayers and nanolayer clusters, in which the Mori-Tanaka method was applied to calculate the modulus of the nanocomposite as a function of various parameters, including the exfoliation ratio, clay layer and cluster aspect ratios, d-spacing, intragallery modulus, matrix modulus and matrix Poisson's ratio [4]. Tsai and Sun investigated the load transfer efficiency of nanoclay platelets in a polymer matrix by using a representative volume element (RVE) and estimated the stress distribution in the RVE using a shear lag model [5]. Wang and Pyrz derived formulas for the overall moduli of composite materials reinforced with transversely isotropic spheroids using the Mori-Tanaka method in view of the morphology of the composites and the anisotropic nature of layered silicate [6]. Sheng et al. presented a multiscale modelling of nanoclay reinforced polymer composites and employed it to analyse the stiffness enhancement mechanisms [7]. Odegard et al. suggested a continuum-based micromechanics model of silica nanoparticle/polyimide composites which incorporated the molecular structures of the nanoparticle, polyimide, and interfacial regions. An effective interface between the polyimide and nanoparticle with properties and dimensions was determined using the results of molecular dynamics simulations [8]. Wang et al. investigated the elastic properties of nano-reinforced polymer composites using a 3D FEM modelling technique [9]. De Guzman and Miravete developed a new micromechanical model denominated Dilute Suspension of Clusters, which considered the heterogeneous dispersion of nano-reinforcement of the composite and the clusters formation [10]

In this study, the effect of microstructural parameters on the elastic properties of composites is investigated. It is found that both the degree of particle clustering and particle's shape have strong effects on the elastic mechanical properties of the composites. This investigation is expected to provide some design parameters for the optimal mechanical behaviors of nano-particle reinforced composites.

\section{FE Modeling of Nano-particle Reinforced Polymer Composites}

\subsection{Representative Volume Element (RVE).}

For numerical analysis of the mechanical behaviors of composites, taking into account its microstructures, representative volume elements (RVEs) of the composites are usually used [9]. The relation of a composite component, multi-particle RVE and unit cell for particle reinforced composites is shown in Fig. 1.

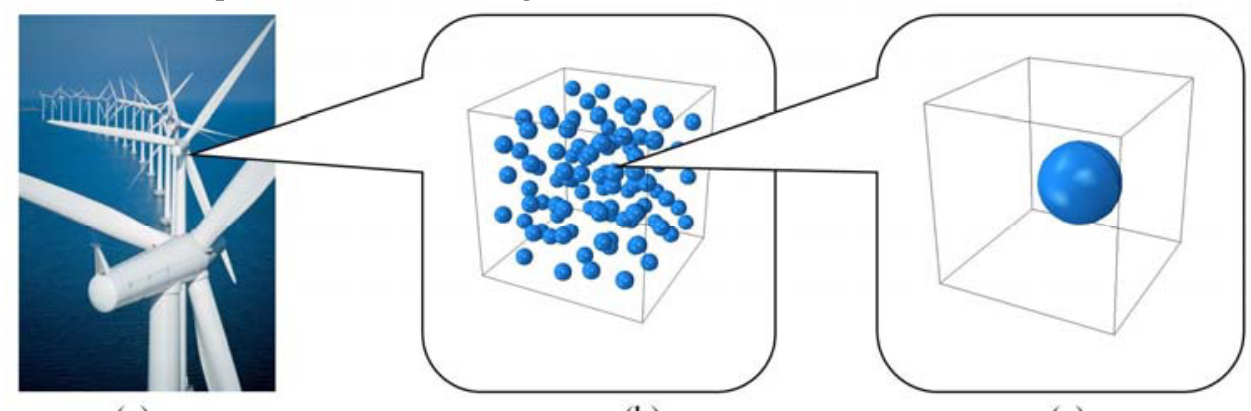

(a)

(b)

(c)

Fig. 1 Illustration among the relation of (a) composite component, (b)multiparticle RVE and (c) unit cell 
Though the Mori-Tanaka model has been used widely to predict the elastic properties of two-phase composites, it is not expected to perform well in nano-scale level because the reinforcement and adjacent polymer region could not be accurately described as consisting of just two phases, i.e., fillers and matrix. To get rid of this drawback, an effective interface model was proposed [8]. This model considered a third phase between the nanoscale reinforcement and matrix, i.e., an effective interface. The assumption employed in this mode includes perfect bonding between different phases (reinforcements, matrix and interface), continuity of the interfacial region, and homogeneity of the interfacial region. Based on these assumptions, the effective interface model is implemented into the FE model of this study. Two kinds of RVEs of with different shaped nano-particles are shown in Fig. 2.

\subsection{Material Properties.}

It is interesting to note that the estimations of the stiffness of the interface layer are quite different in different studies. Some study indicates that the interface layer is softer than the matrix while some study indicates that the interface layer is stiffer than the matrix. In this study, two kinds of interface materials are employed. One is weak interface with Young's modulus of $0.3 \mathrm{GPa}$ and another is strong interface with Young's modulus of $8.4 \mathrm{GPa}$. For the former, the interface corresponds to the functionalized silica composite. For the latter, the Young's modulus of the interface is double than that of the matrix, i.e., 4.2 GPa. The Young's modulus of the fillers is $88.7 \mathrm{GPa}$. The effective particle volume fraction is $5 \%$, and a constant effective interface thickness of $1.2 \mathrm{~nm}$ is adapted [8].

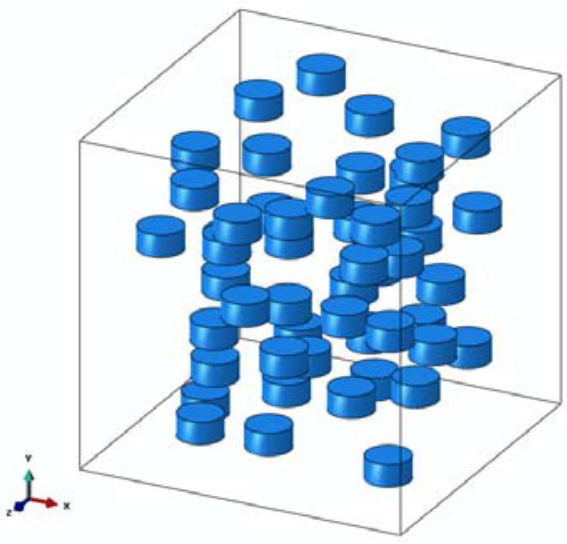

(a) Discal fillers

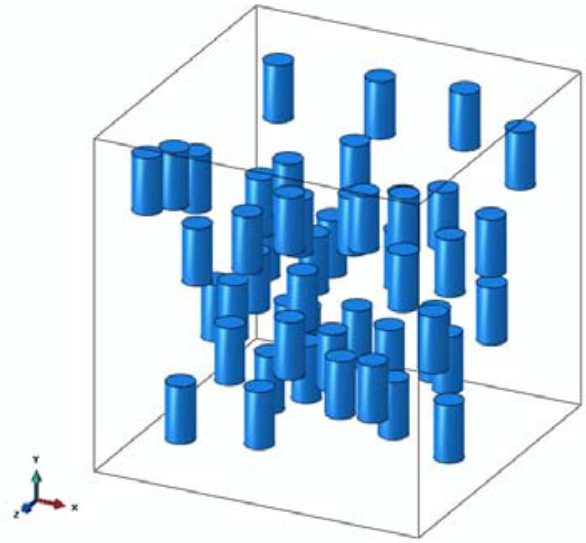

(b) Cylindrical fillers

Fig. 2 Multi-particle RVE models with different shaped fillers

\section{Simulation of Mechanical Behavior}

\subsection{Comparison between single-particle model and multi-particle models.}

In order to confirm the usability of single particle model as a computationally efficient replacement of multi-particle unit cells, we carry out the simulations with unit cell with different particle amount (from 1 to 100) and random arrangements. In all the models, the particle volume fraction is $5 \%$, and the effective interface thickness is $1.2 \mathrm{~nm}$. No interface overlapping is allowed. The results are shown in Fig. 3. 
It can be seen that when only a linear elastic response is considered and no particle clustering occurs, the single particle unit cell approximation is good enough for the analysis of the effect of averaged parameters, like volume contents and size of phases. Apparently, the single particle model cannot be used to analyze the interaction between particles, and multi-particle model must be employed.

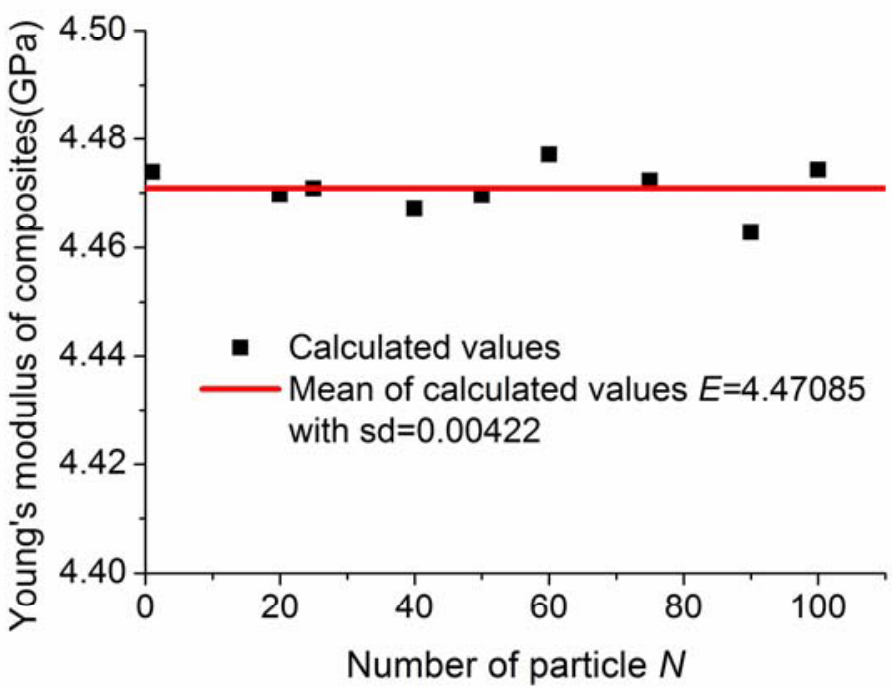

Fig. 3 Young's modulus of RVEs with different number of particles

\subsection{Effect of Particle Clustering.}

The clustering of particles occurs naturally if particles are randomly arranged. Materials with intercalated microstructures represent an important group of the composites. In the clustered structure, the neighboring particles can touch and be located fairly close to another.

In order to characterize the degree of effective interface overlapping, we calculate the coefficient $V / V_{0}$, where $V_{0}$ is the total effective interface volume in a unit cell when there is no interface overlapping, $V$ is the interface volume when some interfaces overlap. When $V / V_{0}=1$, there is no cluster. A small value means that the particles are densely clustered. The relation between Young's modulus of the composite and the degree of the effective interface overlapping is shown in Fig. 4.

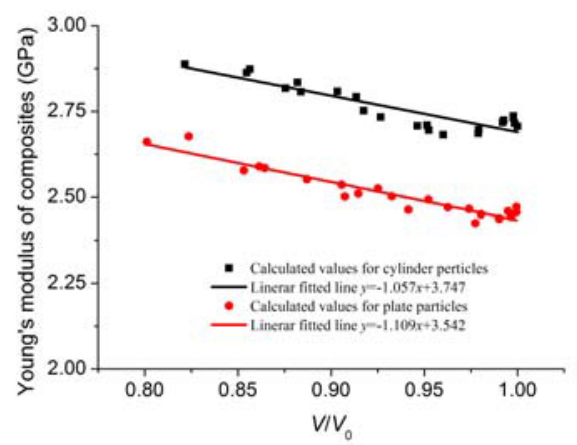

(a) Weak interface 


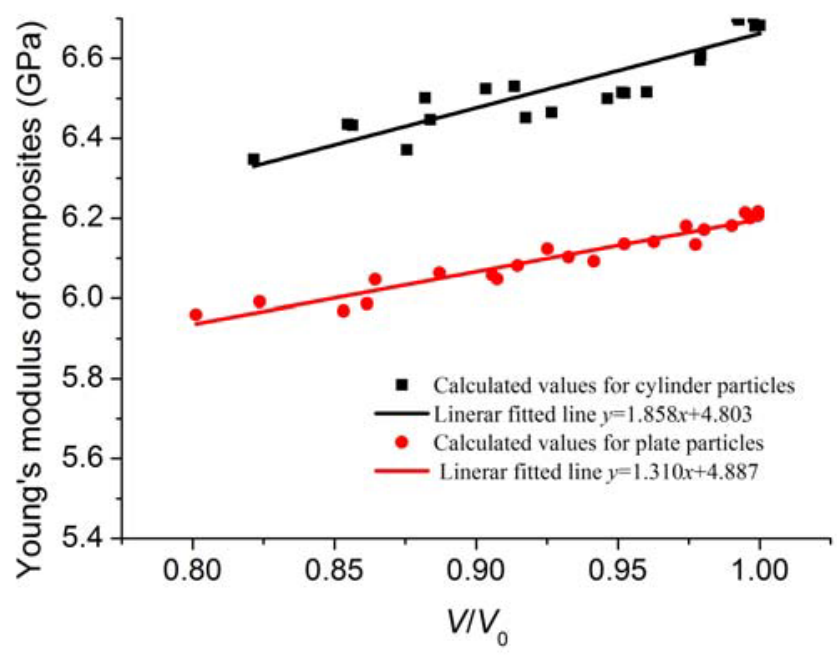

(b) Strong interface

Fig. 4 The relation between Young's modulus and the degree of interphase intersection

It can be seen from Fig. 4 that the degree of the interface overlapping has an effect on the Young's modulus of the composite. More particle clustering means more overlapping of interface layers, and thus lower total volume of interface layers in the unit cell. The softer interface layer is compared to the polymer matrix and particles mean that the particle clustering leads to higher stiffness, and vice versa. In addition, owing to the large volume of interface, the Young's modulus of interface has large effect on that of composites. For example, the Young's modulus of the composites is about 1.5 times than that of matrix for the strong interface case. However, the Young's modulus of the composites is about $60 \%$ of matrix's Young's modulus for the weak interface case. Thus, the manufacturing technology is very important for obtaining appropriate nano-composites. Moreover, one can see from Fig. 4 that the shape of the particles in composites has a strong effect on the elastic properties of the composites. The reinforcement of cylindrical fillers is most effective than discal fillers.

\section{Summary}

Two different shaped particle-reinforced composites are modeled in this paper. Results indicate that the degree of particle clustering and particle's shape have effects on the elastic mechanical properties of composites. The particle clustering leads to higher stiffness for weak interface, and vice versa. The reinforcement of cylindrical fillers is most effective than discal fillers. In addition, single particle unit cell approximation is good enough for the analysis of the effect of averaged parameters when only a linear elastic response is considered without considering the particle clustering in particle-reinforced composites.

\section{Acknowledgements}

The supports of Tianjin Natural Science Foundation (No. 15JCTPJC58800) and National Natural Science Foundation of China (Grant No. 11572223) are greatly acknowledged. 


\section{References}

1. X.L. Ji, J.K. Jing, W. Jiang, B.Z. Jiang, Tensile modulus of polymer nanocomposites, Polym. Eng. Sci. 42 (2002) 983-993.

2. D.A. Brune, J. Bicerano, Micromechanics of nanocomposites: comparison of tensile and compressive elastic moduli, and prediction of effects of incomplete exfoliation and imperfect alignment on modulus, Polym. 43 (2002) 369-387.

3. T.D. Fornes, D.R. Paul, Modeling properties of nylon 6/clay nanocomposites using composite theories, Polym. 44 (2003) 4993-5013.

4. J.J. Luo, I.M. Daniel, Characterization and modeling of mechanical behavior of polymer/clay nanocomposites, Compos. Sci. Technol. 63 (2003) 1607-1616.

5. J. Tsai, C.T. Sun, Effect of platelet dispersion on the load transfer efficiency in nanoclay composites, J. Compos. Mater. 38 (2004) 567-579.

6. J. Wang, R. Pyrz, Prediction of the overall moduli of layered silicate-reinforced nanocomposites - part I: basic theory and formulas, Compos. Sci. Technol. 64 (2004) 925-934.

7. N. Sheng, M.C. Boyce, D.M. Parks, G.C. Rutledge, J.I. Abes, R.E. Cohen, Multiscale micromechanical modeling of polymer/clay nanocomposites and the effective clay particle, Polym. 45 (2004) 487-506.

8. G.M. Odegard, T.C. Clancy, T.S. Gates, Modeling of the mechanical properties of nanoparticle/polymer composites, Polym. 46 (2005) 553-562.

9. H.W. Wang, H.W. Zhou, R.D. Peng, L. Mishnaevsky, Nanoreinforced polymer composites: 3D FEM modeling with effective interface concept, Compos. Sci. Technol. 71 (2011) 980-988.

10. R.G. de Villoria, A. Miravete, Mechanical model to evaluate the effect of the dispersion in nanocomposites, Acta Mater. 55 (2007) 3025-3031. 